\title{
Les langues finno-ougriennes dans la révolution médiatique du « Web 2.0 »
}

The Finno-Ugric languages in the media revolution of Web 2.0

Soome-Ugri keeled Web 2-0 meedia revolutisioon

\section{Sébastien Cagnoli}

\section{(2) OpenEdition}

Journals

Édition électronique

URL : https://journals.openedition.org/efo/437

DOI : 10.4000/efo.437

ISSN : 2275-1947

\section{Éditeur}

INALCO

Édition imprimée

Date de publication : 1 décembre 2012

ISBN : 978-2-343-02592-6

ISSN : 0071-2051

\section{Référence électronique}

Sébastien Cagnoli, «Les langues finno-ougriennes dans la révolution médiatique du « Web 2.0 » », Études finno-ougriennes [En ligne], 44 | 2012, mis en ligne le 22 janvier 2014, consulté le 10 juillet 2021. URL : http://journals.openedition.org/efo/437 ; DOI : https://doi.org/10.4000/efo.437

Ce document a été généré automatiquement le 10 juillet 2021.

\section{(c) (7) (8)}

Études finno-ougriennes est mis à disposition selon les termes de la Licence Creative Commons Attribution - Pas d'Utilisation Commerciale 4.0 International. 


\title{
Les langues finno-ougriennes dans la révolution médiatique du «Web $2.0 »$
}

\author{
The Finno-Ugric languages in the media revolution of Web 2.0 \\ Soome-Ugri keeled Web 2-0 meedia revolutisioon
}

\section{Sébastien Cagnoli}

\section{Introduction : révolutions médiatiques}

1 Deux progrès techniques apparus en Europe au cours $d u \mathrm{Xv}^{\mathrm{e}}$ siècle ont bouleversé le cours de l'Histoire: l'«invention» de l'imprimerie - c'est-à-dire le début de la transmission massive des écrits - et la "découverte » de l'Amérique - c'est-à-dire le début de la navigation intercontinentale à grande échelle. Il ne s'agit nullement de découvertes ou d'inventions absolues, mais leur nouveauté, à cette époque, réside dans le fait qu'elles se déploient soudain dans des proportions jusqu'alors inconcevables ainsi que dans leur simultanéité, qui a multiplié leurs effets. Ces nouveautés ont eu rapidement deux conséquences prodigieuses dans la mesure où elles levaient deux grands obstacles à la communication: d'une part, on pouvait désormais diffuser des informations écrites en masse sans être matériellement limité par la capacité des copistes et des crieurs; d'autre part, l'océan ne constituait plus un obstacle aux déplacements physiques réguliers et à l'échange fréquent d'informations. Il en est donc résulté, au cours des siècles suivants, une accélération exponentielle de la diffusion de l'information et de la communication intercontinentale. Comme avec tous les progrès techniques, les conséquences pouvaient être les meilleures comme les pires, et elles n'ont pas manqué de l'être, du partage des savoirs et des idées jusqu'aux violences et à l'esclavage.

$2 \mathrm{Au} \mathrm{xx}^{\mathrm{e}}$ siècle, l'alphabétisation et les progrès apparus dans le domaine du transport et de la communication ont formé un cadre qui semble tout à fait comparable aux 
événements $\mathrm{du} \mathrm{xv}^{\mathrm{e}}$ siècle rappelés ci-dessus, de par leurs caractéristiques et les premiers effets observables. Là où l'imprimerie faisait naître la transmission massive des écrits, radiotélévision et Internet rendent possible la transmission massive de tous supports textuels et audiovisuels; là où les caravelles pouvaient traverser l'Atlantique, les avions permettent un transport matériel rapide sur toute la surface du globe, pendant que les satellites assurent l'acheminement quasi instantané de toutes les données susceptibles d'être numérisées. L'information textuelle et audiovisuelle peut donc être transmise massivement et instantanément dans la plupart des foyers ${ }^{1}$. Et si tous les foyers n'ont pas d'accès direct à ces informations, tous sont touchés par l'impact qu'ont ces technologies sur l'organisation géopolitique du monde contemporain.

\section{Les langues dans une nouvelle ère de communication}

\section{Le « Web 2.0 »}

3 En particulier, dans les années 2000, le passage au «Web 2.0 » marque une dernière étape, décisive, dans la révolution médiatique amorcée au $\mathrm{xx}^{\mathrm{e}}$ siècle. Que signifie cette expression «Web 2.0 »? Apparue en 2003 avant d'être diffusée par un congrès annuel sur ce thème qui se tient à San Francisco depuis 2005, elle semble s'être généralisée vers 2007. Soulignons qu'elle ne désigne pas une technologie, mais un type de contenu. Pour résumer: il s'agit de pages web dynamiques au contenu personnalisable, interactif, collaboratif, et surtout accessible à tous. Par exemple, il est possible de tenir un blog ou d'utiliser l'ensemble des fonctionnalités de Facebook ou Twitter sans compétence technique particulière - alors que jusqu'au milieu des années 2000, on ne pouvait pas publier grand-chose sur le Web sans se livrer à un minimum de programmation.

Avec la "première version » du Web, l'information était plutôt à sens unique : depuis un émetteur qui maîtrisait l'environnement technique, à destination des lecteurs. À cette époque, les débits étaient lents et les ordinateurs ne se trouvaient pas dans tous les foyers et dans toutes les poches : la diffusion de l'information était limitée. En fin de compte, l'impact n'était pas très différent de celui de la presse ou de la télévision. C'était un moyen alternatif de rechercher des renseignements, une grande encyclopédie, un grand journal et un grand annuaire. Avec le "Web 2.0 », tout le monde devient acteur. La capacité à transmettre massivement de l'information n'est plus entre les mains des seuls professionnels (journalistes, attachés de presse), mais de tous les citoyens. Désormais, chaque individu est potentiellement un émetteur d'information multimédia de masse. Contrairement à l'Internet des années 1990, on n'a plus affaire à une mode ludique ou au charme de la nouveauté, mais bel et bien à une redistribution des pouvoirs. Je ne crois pas que ce soit par légèreté ni par démagogie que Time Magazine, en décembre 2006, ait élu Person of the Year « You » - illustré par un ordinateur multimédia avec une surface réfléchissante en guise d'écran. Après Gandhi, Hitler, De Gaulle ou Khomeini, pour n'en citer que quelques-uns, c'est l'internaute lambda qui, armé des outils du « Web 2.0 » (la couverture contient plusieurs références implicites à YouTube), change le cours de l'Histoire.

5 On l'a vu avec la façon plus ou moins chaotique dont se sont passées les diverses élections qui ont eu lieu dans le monde depuis l'apparition de ce nouveau contexte, 
ainsi qu'avec les manifestations massives et spontanées, voire les révolutions. Les règles du jeu en vigueur (constitutions, lois, codes électoraux...) ne prennent pas en considération ces nouveaux paramètres. Bien souvent, elles ne sont plus pertinentes, dans un environnement où les citoyens s'expriment en temps réel et s'informent de l'actualité les uns les autres avant que les faits soient relatés par des médias ou des organismes accrédités.

Bref, le web collaboratif, en tant qu'outil de communication de masse, échappe au contrôle de masse que l'État pouvait exercer jusque-là. La censure peut toujours être exercée de façon individuelle, mais il est difficile de la mettre en œuvre de façon à la fois automatique et globale... sauf dans le cas particulier où un État décide de fermer certains « robinets » d'Internet. On en a des exemples en Chine, dont les pratiques de censure ont conduit Google à introduire dans ses algorithmes la notion de "mot-clé susceptible de causer des problèmes de connectivité" (Monde 2012); ainsi qu'au Vietnam, avec son «Bamboo Firewall» qui interdit l'accès à un certain nombre de domaines jugés sensibles par les autorités².

\section{Impact sur les langues}

7 Un tel processus d'accélération de la communication internationale à grande échelle a nécessairement un impact sur les langues. Le premier impact auquel on pense, c'est celui du nivellement des idiomes, de leur rapprochement. En effet, les langues du monde s'éloignent quand se dressent des obstacles - peuples de même langue qui migrent sur deux versants d'une chaîne de montagnes ou d'une ligne de partage des eaux (tels les anciens Permiens, qui allaient devenir les Komis et Oudmourtes) ou sur deux rives d'un océan - et elles se rapprochent quand les obstacles diminuent (peuples de langues différentes qui nouent des relations quotidiennes avec des voisins ou des occupants, comme les Mari avec les Tatars, les Finno-Ougriens de Russie avec les Slaves, etc.). Avec Internet, toutes les mers et montagnes du monde s'effacent: chaque individu a la possibilité pratique de communiquer de façon régulière avec chaque individu. À long terme, la tendance à une uniformisation spontanée semble donc inévitable.

8 En contrepartie, toutes les langues sont représentées, et toutes sont visibles, ce qui pourrait renforcer leur prestige, voire leur utilité pratique, et freiner leur disparition (cf. Nikitina 2013). Prenons l'exemple de Wikipedia, l'un des pionniers du « Web 2.0 »: de plus en plus de langues s'y affichent, y compris - et surtout - les langues qui n'ont pas de statut officiel. Pour avoir une première idée (très approximative) de la présence des langues finno-ougriennes sur le Web aujourd'hui, mesurons donc leur activité sur cette encyclopédie collaborative (notamment le nombre d'articles et de participants) ${ }^{3}$ : 


\begin{tabular}{|r|c|r|r|r|r|r|r|}
\hline Rang & Langue & Articles & Pages & $\begin{array}{c}\text { Cumul } \\
\text { éditions } \\
\text { de pages }\end{array}$ & Utilisateurs & $\begin{array}{c}\text { Utilisateurs } \\
\text { actifs }\end{array}$ & Collaborativité $^{4}$ \\
\hline 17 & finnois & 298536 & 805319 & 12301449 & 12301449 & 12301449 & 44 \\
\hline 19 & hongrois & 217974 & 748769 & 205381 & 205381 & 205381 & 97 \\
\hline 41 & estonien & 97118 & 245474 & 1907 & 1907 & 1907 & 30 \\
\hline 141 & $\begin{array}{c}\text { mari des } \\
\text { collines }\end{array}$ & 5063 & 8081 & 12309201 & 12309201 & 12309201 & 3 \\
\hline 142 & võro & 4827 & 8291 & 200272 & 200272 & 200272 & 9 \\
\hline 146 & same du nord & 4270 & 11693 & 2043 & 2043 & 2043 & 7 \\
\hline 153 & komi permiak & 3396 & 7128 & 3184920 & 3184920 & 3184920 & 24 \\
\hline 157 & komi (zyriène) & 3215 & 7640 & 48391 & 48391 & 48391 & 21 \\
\hline 158 & oudmourte & 3080 & 7268 & 508 & 508 & 508 & 44 \\
\hline 160 & $\begin{array}{c}\text { mari des } \\
\text { plaines }\end{array}$ & 3027 & 9411 & 59037 & 59037 & 59037 & 36 \\
\hline 205 & erz'a & 1512 & 3611 & 2106 & 2106 & 2106 & 6 \\
\hline 207 & vepse & 1321 & 2422 & 34 & 34 & 34 & 54 \\
\hline 214 & mokcha & 1117 & 3718 & 150899 & 150899 & 150899 & \\
\hline
\end{tabular}

9 Comme toujours, trois langues occupent une place à part dans la famille : le hongrois, le finnois et l'estonien. En tant que langues officielles de trois États souverains, elles bénéficient d'une visibilité mondiale et d'un emploi quotidien qui ne font que consolider de jour en jour leur stabilité. Dans le paysage du Web mondial, Hongrie, Finlande et Estonie communiquent sur un pied d'égalité avec les autres États souverains (chacune conservant bien sûr ses particularismes). C'est surtout pour les autres langues, celles des peuples non souverains, qu'il va être intéressant d'étudier dans quelle mesure ces nouveaux outils peuvent être utiles.

\section{Les langues finno-ougriennes de peuples non souverains sur les réseaux sociaux}

\section{Présence des langues finno-ougriennes sur le « Web 2.0 »}

10 Ce concept de Web personnalisable et collaboratif a servi de cadre à l'essor des « réseaux sociaux ». Quelle est la place de la famille finno-ougrienne dans cet espace de communication? Des langues minoritaires, en particulier? Il s'agit principalement des langues sames en Norvège, Suède, Finlande et Russie; et de toutes les langues finnoougriennes parlées spécifiquement en Russie.

\section{Les langues sames}

11 Parmi les langues sames, c'est bien sûr celle du nord qui est prépondérante - et la seule qui jouisse d'une certaine notoriété sur Wikipedia, comme on l'a constaté dans le tableau ci-dessus. Les réseaux sociaux permettent de réunir les locuteurs de cette même langue en faisant abstraction des frontières administratives entre Norvège, Suède et Finlande. On ne sera donc pas surpris de retrouver leur vitalité sur Facebook, où des communautés sames se rassemblent sur plusieurs générations.

Du côté de la Russie, les Sames sont beaucoup plus discrets sur le Web, les locuteurs de Kola n'étant estimés qu'à quelques centaines. 


\section{Les langues de Russie}

Pour la Russie, on dispose de statistiques gouvernementales récentes (Perepis 2010), qui fournissent sur les langues les données déclaratives suivantes, que je complète ici avec des observations relatives à la présence sur le web collaboratif :

\begin{tabular}{|l|l|r|r|}
\hline \multicolumn{2}{|c|}{ Langue } & locuteurs & $\begin{array}{c}\text { articles sur } \\
\text { Wikipedia }\end{array}$ \\
\hline \multirow{3}{*}{ mordve } & sans précision & 392941 & \\
\cline { 2 - 3 } & erz'a & 36726 & 1512 \\
\cline { 2 - 3 } & Mokcha & 2025 & 1117 \\
\hline \multirow{3}{*}{ mari } & sans précision & 365127 & \multirow{2}{*}{3027} \\
\cline { 2 - 3 } & des plaines (oriental) & 189 & \\
\cline { 2 - 3 } & des collines & 23062 & 5063 \\
\hline Oudmourte & 324338 & 3080 \\
\hline komi (zyriène) & 156099 & 3215 \\
\hline komi permiak & 63106 & 3396 \\
\hline finnois (non significatif ici) & 38873 & \\
\hline Carélien & 25605 & 291 (en test) \\
\hline Nenètse & 21926 & 49 (en test) \\
\hline estonien (non significatif ici) & 15583 & \\
\hline Khanty & 9584 & 2 (en test) \\
\hline Vepse & 3613 & 1321 \\
\hline Selkoup & 1023 & 4 (en test) \\
\hline Mansi & 938 & 2 (en test) \\
\hline same (non significatif ici) & 353 & \\
\hline Nganassan & 125 & 12 (en test) \\
\hline ingrien & 123 & 70 (en test) \\
\hline Vote & 68 & 245 (en test) \\
\hline enets & 43 & 60 (en test) \\
\hline
\end{tabular}
sur le «Web 2.0 ». L'existence des deux langues erz'a et mokcha continue d'isoler les locuteurs, qui s'identifient par ailleurs d'une façon un peu confuse par rapport à ces deux standards. Comme on le voit dans les deux tableaux ci-dessus, les deux langues sont bien distinguées sur Wikipedia, en même temps que les locuteurs déclarent majoritairement parler «mordve », sans plus de précision. En fin de compte, l'identité mordve est bien visible sur les réseaux sociaux, mais souvent portée par la langue russe.

En langue marie, le portail de Mari-El Radio ${ }^{4}$ est une référence. Ce site d'État fonctionne un peu comme un réseau social, mais avec un flux d'information plus classique, orienté de préférence des journalistes vers les lecteurs. La langue y est très présente et dynamique. De même, le site d'information MariUver $^{5}$ (en russe, mari, estonien et 
anglais) est très visible sur le Web, au moyen de vastes réseaux de contacts dans tout l'espace finno-ougrien et d'une présence active sur plusieurs sites à forte fréquentation (Twitter, Facebook, YouTube).

18 Dans le domaine oudmourte, la langue est très vivante sur les groupes et sur les pages individuelles. Le groupe Facebook «Udmurt sjamen » et la chaîne YouTube d'olga Urasinova en sont deux exemples parmi tant d'autres. En outre, on peut signaler l'existence d'un petit groupe bessermane sur VKontakte («МИ БЕСЕРМАНъЁС»), qui ne rencontre pas un grand succès; en dépit du nom, les échanges s'y font plutôt en russe.

Quant aux langues komies: sur les réseaux sociaux, les échanges peuvent se dérouler en komi et en permiak, les deux langues étant suffisamment proches pour que leurs spécificités ne soient pas un obstacle à la conversation, à plus forte raison à l'écrit. Le particularisme de l'Ižma n'est pas spécialement mis en valeur. Les différentes langues et dialectes komis sont trop proches - et souvent maniés de façon trop approximative pour que leur différentiation soit pertinente. L'utilisation du «Web 2.0 » est quotidienne dans les villages, et elle est bien sûr d'autant plus généralisée dans les villes universitaires.

\section{Utilisation des réseaux sociaux}

\section{Deux réseaux sociaux concurrents}

Créé en 2006 par le Pétersbourgeois Pavel Durov, qui était alors un jeune diplômé de vingt-deux ans, VKontakte est devenu très vite un site incontournable en Russie et dans quelques pays voisins (surtout Biélorussie, Ukraine et Kazakhstan). L'interface, qui s'inspirait nettement de celle de Facebook, n'était disponible qu'en russe jusqu'en octobre 2009.

21 Le succès de VKontakte auprès des internautes russophones s'explique par le fait que Facebook n'offrait alors, et jusqu'à juin 2008, qu'une interface en anglais. Les premiers internautes de Russie à y apparaître étaient surtout des gens qui avaient tissé un réseau social lors d'un séjour aux États-Unis ou en Europe.

Depuis 2009, grâce à leurs interfaces multilingues, les deux sites concurrents sont maintenant accessibles à un public plus vaste. En particulier, on a pu observer récemment une forte augmentation d'utilisateurs de Russie sur Facebook (« le grand public », et non plus seulement les étudiants cosmopolites). Dans le monde finnoougrien, ces deux outils s'avèrent complémentaires.

Il serait vain de chercher à énumérer ici les groupes d'intérêt finno-ougrien essaimant sur ces réseaux tant ils sont nombreux ; et l'on peut sans peine les parcourir en suivant les liens des utilisateurs et des groupes. Si la langue nationale est utilisée comme signe de reconnaissance, elle se limite souvent aux salutations ou à quelques expressions idiomatiques (comme c'est souvent le cas en France sur les sites régionaux), pour céder rapidement la place au russe. Néanmoins, certains utilisateurs et certains groupes manient le bilinguisme avec beaucoup de naturel et savent s'exprimer principalement dans leur langue finno-ougrienne tout en passant au russe quand le contexte (sujet, source, destinataires) le justifie.

Outre les rencontres et la conversation - qui jouent déjà un rôle essentiel dans le développement des langues - la structure du site permet la diffusion d'informations relatives aux événements culturels, aux questions politiques et sociales, à l'actualité de 
la recherche. Certains membres sont plutôt auteurs - les plus actifs sont omniprésents, nouent des contacts personnels avec tous leurs lecteurs, créent et diffusent des néologismes...; d'autres sont plutôt lecteurs - et les flux d'information de ces réseaux constituent donc une alternative sérieuse aux médias traditionnels.

Les autres réseaux sociaux concurrents ont une importance moindre, mais on peut mentionner aussi Orkut et Google+ (tous deux affiliés aujourd'hui à Google), où s'expriment occasionnellement quelques défenseurs des langues finno-ougriennes minorées - souvent les mêmes personnes, qui cherchent à être visibles sur un maximum de canaux médiatiques.

\section{Sur les autres sites}

Parmi les nombreux moyens de diffusion des flux Twitter, il faut souligner qu'il existe la possibilité, couramment utilisée, de relayer automatiquement les messages sur Facebook ou VKontakte. En outre, rappelons que la plupart des sites d'information et blogs fournissent aussi leur contenu sous forme de flux (généralement dans un format RSS) mis à la disposition des autres sites web. En effet, l'une des caractéristiques du «Web 2.0 » est la syndication de contenu, c'est-à-dire la possibilité de construire des pages dont le contenu est issu en temps réel de différents sites. Bien souvent, il suffit donc d'être inscrit sur un seul site pour pouvoir bénéficier des informations fournies par les autres.

Les chansons en langues finno-ougriennes - traditionnelles ou d'artistes - circulent largement. De même, sur YouTube (mais aussi et surtout sur VKontakte, moins contrôlé sur les questions de propriété intellectuelle), de nombreux films sont partagés. Par exemple, deux longs métrages en langue oudmourte sont diffusés sur des sites «Web 2.0»: le film de 1994 L'ombre d'Alangasar (АЛАНГАСАРЛЭН ВУЖЕРЕЗ / ТЕНЬ АЛАНГАСАРА, 1994) ${ }^{6}$ et Les fraises (УзЫ-БОРЫ) ${ }^{7}$. Enfin, on voit aussi passer des expériences moins professionnelles, mais tout aussi originales, comme des doublages komis de dessins animés soviétiques (c'est-à-dire "de notre enfance») ou de séquences de grands succès mondiaux du cinéma contemporain (Harry Potter ${ }^{8}$ ).

Parmi les sites qui se sont épanouis ces dernières années, il faut souligner l'existence d'une invention purement finno-ougrienne, que l'on doit à Artëm Malyh (Ortem Jorgi, Jorgi Ortjomi), étudiant oudmourte à Moscou. Son portail Uralistica, créé en juin 2008, présente toutes les caractéristiques d'un réseau social, auxquelles s'ajoute un service de blogs. Uralistica a pour vocation de réunir des individus du monde entier personnellement impliqués dans les affaires finno-ougriennes par un engagement socioculturel ou franchement politique ${ }^{9}$. De fait, Uralistica est devenu rapidement le site le plus actif du «Web 2.0 » finno-ougrien. L'oudmourte et les langues mordves y couvrent environ $12 \%$ des communications (avec une dominance assez nette de l'erz'a sur le mokcha).

29 Une autre invention oudmourte (créée depuis Kazan, semble-t-il) est apparue en 2012 avec le nom de domaine Yaraton.ru. Elle se présente comme un «УДМУРТскИЙ САЙТ ЗНАКОмСтВ», "site oudmourte de rencontres » : chaque membre y indique son sexe, celui des personnes cherchées, et ce qu'il attend de ces rencontres ("amitié, amour, romance, sexe, mariage ou autre »). L'interface est en russe, mais le nom du site est en oudmourte (ЯРАТОН = amour), ainsi que l'épigraphe, refrain d'une chanson populaire. Une partie des membres s'y présentent en langue oudmourte. Avec 229 inscrits (au 
20 juin 2012), l'initiative est loin d'être un succès phénoménal, mais la démarche est significative.

Tandis que de jeunes Oudmourtes encouragent les internautes finno-ougriens à communiquer, et à partager leurs idées et leur créativité, la situation est très différente chez les Komis. À Syktyvkar, c'est l'État qui a lancé des initiatives finno-ougriennes sur le «Web $2.0 »$ : le portail Finugor.ru ${ }^{10}$ (rédacteur en chef: Jurij Popov), créé dans le cadre du Congrès des peuples finno-ougriens; Finnougoria ${ }^{11}$, ouvert en 2007 comme portail du Centre culturel finno-ougrien fédéral de Russie (qui a son siège à Syktyvkar) ; et Finnougr.ru ${ }^{12}$ (rédactrice en chef : Polina Romanova) depuis 2008. Ces sites ne sont pas du tout en concurrence avec les réseaux sociaux (encore que ce dernier fournisse des services assez détaillés de profil personnel, de recherche de contact et de réseau de connaissances, un peu comme le portail de Mari-El Radio) : ils ont surtout une mission d'information (gouvernementale), et leur interactivité se limite généralement à la possibilité de poster des articles et des commentaires. On retrouve là une constante chez les peuples de langues permiennes: en Komi, c'est principalement l'État qui défend les langues et cultures finno-ougriennes; en Oudmourtie, ce sont essentiellement des initiatives privées (associatives ou individuelles) ${ }^{13}$.

L'ADÉFO n'est pas en reste, puisqu'elle est présente sur YouTube ${ }^{14}$, où sont publiés des matériaux audio et vidéo (colloques scientifiques, lectures de poésie, débats), ainsi que sur Facebook ${ }^{15}$, fut-ce discrètement. De même, les chercheurs de l'université de Vienne sont très actifs. Mais ce sont surtout les locuteurs natifs, bien sûr, qui font vivre leurs langues sur le Web.

\section{« L'internationale finno-ougrienne »}

\section{L'identité ressuscitée}

32 Je suis tenté de qualifier de «néo-mérien » le mouvement identitaire qui apparaît dans la région de l'oka et de la haute Volga, territoire des anciens Mériens, Mouromiens et Mechtchériens, dont les langues se sont éteintes au Moyen-Âge avant d'avoir pu être documentées. L'existence, aujourd'hui, d'une identité mérienne significative a fait l'objet d'un film intitulé Le dernier voyage de Тапуа (овсянки), réalisé par Alekseï Fedortchenko en 2010 et présenté notamment à Venise. La notion de « Merjamaa » - en caractères latins, y compris dans un contexte en russe - semble avoir le vent en poupe, comme le suggèrent le portail Merjamaa ${ }^{16}$ et le groupe VKontake « MERJAMAA - ЗЕМлЯ MEPЯ » (le modèle finnois n'est pas loin).

33 La langue prend ici une position secondaire : les défenseurs de l'identité mérienne sont russophones, et les revendications nationales sont d'une nature qui n'est pas linguistique ${ }^{17}$. Néanmoins, ce mouvement a une visibilité suffisamment importante pour mériter d'être mentionné, d'autant plus qu'il fait partie intégrante de ce qu'on pourrait qualifier d'«internationale finno-ougrienne» (de par sa participation à Uralistica et aux divers réseaux sociaux mentionnés précédemment).

\section{La solidarité finno-ougrienne}

34 Le portail Uralistica cherche à fédérer une population "finno-ougrienne » en s'affranchissant des frontières linguistiques et administratives. De même, les groupes Facebook et VKontakte sont largement utilisés pour réunir des communautés "pan- 
finno-ougriennes ». On pouvait déjà procéder à ce genre de regroupement dans les années 1990 sur les forums et les listes de diffusion, certes; mais le "Web 2.0 », en apportant plus de souplesse et de spontanéité, augmente de façon décisive la vitesse et l'étendue de la diffusion des messages.

Même en parcourant les différentes pages consacrées à des langues et cultures spécifiques, on remarque une forte "solidarité finno-ougrienne ». En voici un exemple récent et évocateur. Lors du grand succès populaire des mémés oudmourtes de Buranovo, finalistes de l'Eurovision en 2012 sous les couleurs de la Russie, on a pu observer des messages de soutien et des relais d'information sur la plupart des sites évoqués ci-dessus, non seulement sur les sites oudmourtes et globalement finnoougriens, mais aussi et surtout sur les sites consacrés à d'autres spécialités finnoougriennes. En voici un, relevé le 22 mai 2012 sur le groupe Facebook « коми кыв » :

Si les Oudmourtes gagnent, ce sont tous les Finno-Ougriens qui gagneront ${ }^{18}$ !

\section{Libertés et limites}

\section{Obstacles techniques et solutions}

36 J'ai été surpris en constatant qu'un ami avec lequel je parle komi m'écrivait systématiquement en russe sur VKontakte. Bien que le komi soit la langue quotidienne en usage dans son village, il m'a expliqué que, devant le clavier, l'obstacle des caractères spéciaux ( $i, I, \ddot{o}, \ddot{O})$ est tel qu'il lui est plus facile, plus naturel d'écrire en russe. Ainsi, pour des internautes bilingues qui sont à l'aise en russe, qui utilisent du matériel russe (clavier, logiciels), il est plus facile d'écrire dans la langue de la fédération que dans celle de la famille. Il faut dire aussi que le russe a été appris de façon rigoureuse à l'école, tandis que le komi est souvent une langue parlée couramment, mais dont les règles d'orthographes sont négligées, voire méconnues, ce qui rend son usage écrit d'autant plus laborieux.

37 Le fait d'écrire dans une langue finno-ougrienne de Russie sur des sites collaboratifs dénote donc une véritable volonté, presque un combat: on fait l'effort de surmonter les difficultés techniques - en les contournant ou en cherchant à les résoudre - et de posséder une certaine maîtrise de la langue. Et de fait, malgré ces obstacles qui incitent manifestement de nombreux internautes à opter pour la langue russe, on constate que les langues minorées fleurissent sur les réseaux sociaux.

Les solutions à ces problèmes sont diverses, et rarement tout à fait satisfaisantes. Par exemple, le caractère latin $\ddot{0}[00 \mathrm{~F} 6]$, peut être utilisé à la place du caractère cyrillique $\ddot{O}$ [04E7]; mais ce n'est qu'un palliatif, qui devient apparent et handicapant lorsqu'on procède à des tris alphabétiques automatiques. Sur ces questions techniques, de nombreuses personnes se penchent depuis longtemps. À présent, l'utilisation des caractères spéciaux est possible de manière standard grâce au codage Unicode sur deux voire trois ou quatre octets (UTF-8). On dispose même ainsi de l'alphabet komi de Molodcov (dans la plage complémentaire à l'alphabet cyrillique de 0500 à 052F)... et d'un projet de normalisation du codage de l'ancien alphabet de saint Étienne de Permie. Mais tout cela reste difficile d'accès au clavier.

Comme solution radicale au problème des caractères spéciaux, on voit apparaitre des tentatives de latinisation. L'objectif n'est pas d'élaborer un nouvel alphabet linguistiquement pertinent, mais seulement d'adopter une table de translittération qui 
permette d'écrire facilement sa langue avec les moyens dont on dispose - à savoir un clavier russe-anglais standard. Ainsi, sur le groupe VKontakte «коми культурА ДА коми кыв МосквАЫн!» (« La cULTURE комIE et la LANGUE кOMIE à Moscou!»), Oleg Motorin utilise une translittération de son cru, qui consiste à faire correspondre à chaque lettre cyrillique une lettre latine facile d'accès sur le clavier - avec des idées assez saugrenues, comme $w$ pour $\omega$. Au-delà de son caractère résolument pragmatique, ce palliatif semble dénoter aussi une certaine volonté de se détacher de l'influence russe et de se rapprocher intellectuellement des cousins fenniques. Sans surprise, on remarque que ces expériences vont de pair avec la proposition d'un nouveau drapeau komi, qui jouit d'une certaine popularité sur les réseaux sociaux : les trois couleurs y sont disposées non pas en bandes verticales - le drapeau komi officiel étant de ce point de vue calqué sur le drapeau russe - mais en croix scandinave comme sur les drapeaux des Finlandais et des peuples fenniques de Russie.

De même, il n'est pas rare de voir le mokcha écrit en alphabet latin, par exemple sur le groupe VKontakte «Mо К ̧̧ $\mathrm{E}$ ». Cet usage fait penser au cas du tatar, dont l'écriture officielle repose sur l'alphabet cyrillique, mais qui se répand aussi sur le Web avec l'alphabet latin - pour des raisons d'abord pratiques, mais probablement aussi plus ou moins idéologiques, l'alphabet latin étant officiel pour d'autres langues turciques hors de Russie.

Par ailleurs, de plus en plus de langues sont proposées pour les interfaces des systèmes d'exploitation et des logiciels. VKontakte est actuellement disponible en erz'a, oudmourte et mari. Du côté de Facebook, il faut attendre encore. Cela dit, un bilinguisme équilibré permet d'utiliser un logiciel écrit dans une langue et de s'y exprimer dans une autre (personnellement, j'utilise une multitude de logiciels dont les menus sont en anglais, ce qui ne me paraît pas présenter de menace pour mon confort ni pour la vitalité de mon français). La question de l'interface est donc plus symbolique que pratique.

Pour encourager l'usage des langues, de nombreux logiciels ont été développés ces dernières années - notamment des outils en ligne, plus adaptés à la logique générale du «Web 2.0»: fontes, analyseurs morphologiques, dictionnaires, supports d'apprentissage... En particulier, les équipes de l'Université de Vienne et de Mari El Radio réalisent en collaboration des outils sophistiqués pour faciliter l'apprentissage et l'usage du mari (dictionnaire, analyseur morphologique) ${ }^{19}$. Un vaste projet porte déjà ses fruits, celui d'un " guide de conversation finno-ougrien » multilingue ${ }^{20}$ (développé à l'initiative de Finugor.ru et financé par la République de Komi), qui offre toutes les correspondances entre deux langues de grande diffusion (le russe et l'anglais) et les langues finno-ougriennes. Chacun en fera l'usage qui lui conviendra, que ce soit pour apprendre à communiquer à l'oral, pour mener des conversations en ligne, ou ne serait-ce que pour saluer ses amis internautes mokchas, à l'heure de passer à table, d'un «ТА́НЦТИНЯСТА ЯРХЦАК».

\section{Espoirs et limites des libertés du Web}

La «Fondation pour la défense de la glasnost" (Фонд ЗАщиты ГЛАсности), organisation non gouvernementale fondée en 1991 à Moscou, met à jour régulièrement une carte rendant compte de la liberté des médias (imprimés et électroniques) (GDF 2012). Les sujets de la fédération y sont répartis en quatre catégories, en fonction 
de l'évaluation qui a été faite au cours de la période considérée. La dernière version disponible date d'avril 2010 (mesures réalisées entre mars 2009 et février 2010).

Un critère est décisif dans cette classification: celui du monopole d'État. Le fait qu'il n'existe pas de médias privés range automatiquement les régions concernées dans «relativement non libre ». Si des pressions ou des violences sont observées, on passe dans la catégorie «non libre ». La position des républiques d'Oudmourtie et de Komi et des territoires de Nénetsie et d'Ougrie est stable : «relativement non libres ». Mari-El, Mordovie et Iamalie sont présentés comme «non libres ». Et la Carélie, depuis 2008, est passée de «non libre » à « relativement libre ».

Dans un pays où la presse est un monopole de l'État et où certains journalistes peuvent être exposés à des violences physiques, la révolution $\mathrm{du}$ « Web 2.0 » offre une véritable alternative à la liberté de communiquer.

Par ailleurs, les libertés d'association et de réunion sont généralement encadrées par des lois. Pourtant, sur les réseaux sociaux, aujourd'hui, il est possible de se rencontrer et de se " réunir " (d'une part en créant un réseau de contacts personnels au premier degré, d'autre part en adhérant volontairement à des communautés) sans considération de frontières et sans déclaration préalable à la police : la réunion est d'abord virtuelle, certes, mais le potentiel d'échange d'idées et de réalisation de projets collectifs est à peu près aussi vaste que dans le cas d'une association encadrée par exemple par la loi française de 1901 - à la seule exception, non négligeable, certes, de la question financière.

Un exemple concret de ces libertés a été apporté récemment par Pavel Durov. Au cours des mouvements de contestation consécutifs aux élections à la Douma, début décembre 2011, il signale que VKontakte subit régulièrement des tentatives de pression lui demandant de supprimer des serveurs de VKontakte certaines pages " indésirables ", notamment des groupes ou profils d'opposition. Il refuse obstinément de s'y soumettre et rappelle sa profession de foi (le 6 décembre, sur son flux Twitter) :

Je ne peux soutenir ni l'opposition, ni le pouvoir, ni aucun parti politique.

VKontakte est une organisation $100 \%$ apolitique ${ }^{21}$.

Le 7 décembre, il publie sur Twitter ${ }^{22}$ et VKontakte ${ }^{23}$ une lettre officielle du FSB (datée du jour même $)^{24}$ lui demandant de fermer un certain nombre de groupes et rendez-vous du serveur vkontakte.ru, à laquelle il répond officiellement par la photo d'un chien tirant la langue. La seule censure à laquelle il consente à se soumettre, c'est la vérification interne du respect des conditions d'utilisation du site (pas de harcèlement, pas d'incitation à la haine, etc.); mais la diversité des idées et l'organisation de rassemblements pacifiques sont parfaitement compatibles avec les valeurs de VKontakte, qui se présente ainsi comme une véritable incarnation des espoirs de liberté d'expression en Russie.

D'une manière générale, il est évident que tous les sites «Web 2.0 " sont largement exploités par les services de renseignement, afin de se tenir informés des idées exprimées par les citoyens et de leur impact possible sur les masses. On vient d'en voir un exemple relatif aux élections législatives, mais tous les débats de société sont susceptibles d'être surveillés - et les questions nationales ne font certainement pas exception. 


\section{Conclusion : une nouvelle répartition} peu une nouvelle répartition des personnes, l'émergence de nouvelles identités, la formation de nouvelles communautés, sur d'autres critères, notamment linguistique. On pourrait dire qu'il appartient aux citoyens, dans ce contexte, de redéfinir l'idée de «nation».

Quelle que soit l'évolution future du Web, quelles que soient les nouvelles technologies à venir, il me semble que le cap franchi dans les années 2000 avec le pouvoir donné à chaque individu de s'adresser en temps réel à tous ses semblables ouvre une nouvelle ère pour les peuples et pour les langues. Vers plus de libertés et vers plus d'oppression, vers de plus grands accomplissements et de plus grands échecs... vers de nouveaux espoirs, parfois satisfaits, parfois déçus... bref, vers de nouvelles pages de l'histoire de l'humanité.

\section{BIBLIOGRAPHIE}

\section{Presse}

Lenta 2011 = «ПАВЛА ДУРОВА ВЫЗВАЛИ В ПРОКУРАТУРУ» // ЛЕНТА.РУ, 09.12.2011, 19 :34 :09 [page consultée le 18 juin 2012] : http://lenta.ru/news/2011/12/09/prosecutor

Monde 2012 = «Censure : Google modifie son moteur de recherche en Chine » // Le Monde, $1^{\mathrm{er}}$ juin 2012 [page consultée le 8 juin 2012] : http://www.lemonde.fr/technologies/article/ 2012/06/01/censure-google-modifie-son-moteur-de-recherche-en-chine_1711056_651865.html.

Sites d'information

GDF 2012 = ФоНД ЗАщИТы ГЛАСНости / Glasnost Defence Foundation, page consultée le 8 juin 2012 : http://www.gdf.ru/map

IWS 2012 = Internet World Stats, Miniwatts Marketing Group, pages consultées le 8 juin 2012 : http://www.internetworldstats.com/surfing.htm, http://www.internetworldstats.com/ stats.htm, http://www.internetworldstats.com/top20.htm

Perepis 2010 = ВСЕРОССИЙСКАЯ ПЕРЕПИСЬ НАСЕЛЕНИЯ 2010 ГОДА, ФЕДЕРАЛЬНАЯ СЛУЖБА ГОСУДАРСТВЕННОЙ СТАТИСТИКИ [Russie], 2009-2012 : http://www.perepis-2010.ru/ RSF 2012 = Reporters sans frontières, page consultée le 8 juin 2012 : http://march12.rsf.org/fr/ Sites web 2.0

Facebook= https://www.facebook.com/

Uralistica $=$ http://uralistica.com/

VKontakte $=$ http://vk.com 


\section{NOTES}

1. Selon le site Internet World Stats (alimenté par la société Miniwatts), au 31 mars 2012, les « internautes » constituent $32,7 \%$ de la population mondiale. Le pourcentage s'élève au-dessus de $60 \%$ si l'on réduit le périmètre à l'Europe, à l'Amérique du Nord ou à l'Océanie. Les internautes les plus nombreux résident, par ordre décroissant, en Chine, États-Unis, Inde, Japon, Brésil, Allemagne, Russie, Indonésie, Royaume-Uni, France, etc. (IWS 2012).

2. Ce ne sont là que deux exemple parmi bien d'autres. «Reporters sans frontières " a établi en 2011 et 2012 des cartes de la « cyber-censure » (RSF 2012). La liste est courte, et l'on peut donc citer ici exhaustivement, d'une part, les pays déclarés « sous surveillance » : France et Russie, aux côtés d'Australie, Corée du Sud, Égypte, Émirats arabes unis, Érythrée, Inde, Kazakhstan, Malaisie, Sri Lanka, Thailande, Tunisie, Turquie ; d'autre part, les « ennemis d'Internet »: Arabie saoudite, Bahreïn, Biélorussie, Birmanie, Chine, Corée du Nord, Cuba, Iran, Ouzbékistan, Syrie, Turkménistan et Vietnam.

3. http://meta.wikimedia.org/wiki/List_of_Wikipedias, statistiques du 6 juin 2012 à 00:29 UTC (page consultée le 8 juin 2012).

4. http://mari-el-radio.ru/.

5. http://mariuver.wordpress.com/ et http://mariuver.info/

6. http://vimeo.com/20788076

7. http://youtu.be/Vu_LW_2RBIs

8. «ГАРРИ ПОТТЕР НА КОМИ ЯЗЫКЕ».

9. Le site est décrit comme ceci sur sa page d'accueil: «Finno-Ugric social network for collaboration and action. ФИННО-УГОРСКАЯ СОЦИАЛЬНАЯ СЕТЬ ИССЛЕДОВАТЕЛЕЙ, АКТИВИСТОВ и политиков».

10. http://finugor.ru/

11. http://www.finnougoria.ru/

12. http://www.finnougr.ru/

13. Il faut se rappeler que le centre économique et culturel, dans les deux cas, est la capitale de la république : or Syktyvkar est historiquement une ville commerçante de tradition komie, tandis qu'Iževsk a toujours été une ville industrielle russe.

14. http://www.youtube.com/user/AdefoParis

15. http://www.facebook.com/AdefoParis

16. http://merjamaa.ucoz.ru/

17. Il est important de souligner que le site insiste sur le fait que ses intentions ne sont pas politiques, mais strictement culturelles, et qu'il invite au dialogue (http://merjamaa.ru/index/ 0-2, page consultée le 19 juin 2012).

18. «ВЕРМАСНЫ КӦ УДМУРТЪЯС - ВЕРМАСНЫ ЖӦ ФИНН-ЙӦГРАЯС!»

19. http://www.univie.ac.at/maridict/

20. http://www.fulib.ru/phrasebook/

21. «Я НЕ МОГУ ПОДДЕРЖИВАТЬ НИ ОППОЗИЦИЮ, НИ ВЛАСТЬ, НИ КАКУЮ-ЛИБО ПАРТИЮ. ВКОНТАКТЕ - 100 \% АПОЛИТИЧНАЯ ОРГАНИЗАЦИЯ.»

22. https://twitter.com/durov

23. http://vk.com/durov

24. http://twitpic.com/7q9u8o (page consultée le 18 juin 2012). 


\section{RÉSUMÉS}

La révolution médiatique d'Internet, et plus particulièrement du «Web 2.0 » dans les années 2000, a entamé une modification radicale des moyens de communication entre les citoyens du monde. Quelle est la place des langues finno-ougriennes dans cet espace d'échange? En quoi cet outil peut-il être utile aux peuples de langues finno-ougriennes, en particulier aux peuples non souverains? Quelles sont ses perspectives et ses limites? Cette réflexion s'appuie sur les sites web considérés sous leur forme actuelle (juin 2012) et sur la presse récente.

The media revolution of the Internet, especially the so-called "Web 2.0" in the 2000s, have fundamentally changed the means of communication between citizens of the world. What is the role of Finno-Ugric languages in this space of exchange? In what way can this tool be useful to the Finno-Ugric peoples, especially the non-sovereign ones? What are its prospects and limitations? This reflection is based on the "Web 2.0" websites in their present form (June 2012) and on recent press.

Meedia revolutsioon internetis, iseäranis käesoleva sajandi algusaastatel levima hakanud Web 2.0 on põhjalikult muutnud inimeste suhtlemisviise kogu maailmas. Milline on soome-ugri keelte positsioon selles uues suhtlusdimensioonis? Kuidas saab Web 2.0 olla kasuks soome-ugri rahvastele, eeskätt neile, kel puudub omariiklus? Millised on selle perspektiivid ja piirangud mainitud kontekstis? Siinne käsitlus põhineb 2012. aasta juunikuu seisuga Web 2.0 võimalusi kasutanud kodulehekülgedel ning uuema aja ajakirjandusel.

\section{INDEX}

Mots-clés : Internet, Web 2.0, réseaux sociaux, alphabet Molodcov, Vkontakte, Facebook, Twitter, YouTube, Wikipédia, Mari Uver, Orkut, Google+, Uralistica, Finnougorija, Eurovision Keywords : Internet, Social networks, Media, Freedom of expression, Censorship Index chronologique : XXIe siècle (début), XXIe siècle Index géographique : Hongrie, Finlande, Estonie, Norvège, Suède, Fédération de Russie, France, Buranovo, Iževsk, Syktyvkar, Khanty-Mansiïsk (Ougrie) (district autonome), Oudmourtie (République), Komi (République), Iamalo-Nénétsie (Yamalie) (district autonome), Mordovie (République), Carélie (République)

Thèmes : sociolinguistique

disciplines finnois, erza, mari des collines, võro, same du Nord, komi-permiak, mari des plaines, komi, oudmourte, mokcha, vepse, khanty, nénetse, selkoupe, carélien, nganassan, vote, bessermane, mérien 